\title{
A quantum version of Wielandt's inequality
}

\author{
Mikel Sanz, David Pérez-García, Michael M. Wolf, and Juan I. Cirac
}

\begin{abstract}
In this paper, Wielandt's inequality for classical channels is extended to quantum channels. That is, an upper bound to the number of times a channel must be applied, so that it maps any density operator to one with full rank, is found. Using this bound, dichotomy theorems for the zero-error capacity of quantum channels and for the Matrix Product State (MPS) dimension of ground states of frustration-free Hamiltonians are derived. The obtained inequalities also imply new bounds on the required interaction-range of Hamiltonians with unique MPS ground state.
\end{abstract}

Index Terms-classical channels, information rates, quantum channels, spin systems, strongly correlated electrons, Wielandt's inequality.

\section{INTRODUCTION}

$\mathbf{C}$ ONSIDER a classical memoryless channel acting in discrete time on an alphabet of size $D$. Such a channel is described by a stochastic matrix $A \in \mathcal{M}_{D \times D}$ which is called primitive [1] if there is an $n \in \mathbb{N}$ such that $\left(A^{n}\right)_{i, j}>0$ for all $i, j$. The minimum $n$ for which this occurs, $p(A)$, is called the (classical) index of primitivity of $A$ (or the exponent of $A$ ). This ensures that after applying the channel $p(A)$ times to any probability distribution, there will be a non-zero probability for any possible event. Wielandt's inequality [2] states that, for every primitive matrix,

$$
p(A) \leq D^{2}-2 D+2
$$

and this is the optimal bound which is independent of the matrix elements. Wielandt's inequality has a wide range of applications in different fields, ranging from Markov chains [3] to graph theory and number theory [4], and numerical analysis [5].

In this work, we derive a quantum analogue of Wielandt's inequality. That is, we consider quantum channels, i.e., trace preserving completely positive linear maps (TPCPM) and define a property analogous to primitivity: the existence of an $n \in \mathbb{N}$ such that after the $n$-fold application of the quantum channel every positive semidefinite operator is mapped onto a positive definite operator. The smallest such $n$ then defines a quantum index of primitivity, $q$. We begin by relating primitivity to some other properties of quantum channels, such

Manuscript received September 30, 2009; revised .

M. Sanz and J. I. Cirac acknowledge the support of the QCCC Program of the EliteNetzWerk Bayern and the DFG (project FOR635), D. PérezGarcía acknowledges the support of the Spanish grants I-MATH, MTM200801366, S2009/ESP-1594 and the EU project QUEVADIS and M. M. Wolf acknowledges support by QUANTOP, the Danish Natural Science Research Council (FNU) and the EU projects QUEVADIS and COQUIT.

M. Sanz and J. I. Cirac are with the Max-Planck-Institut für Quantenoptik, Hans-Kopfermann-Str. 1, 85748 Garching, Germany.

D. Pérez-García is with the Dpto. Análisis Matemático and IMI, Universidad Complutense de Madrid, 28040 Madrid, Spain.

M. M. Wolf is with the Niels Bohr Institute, Blegdamsvej 17, 2100 Copenhagen, Denmark. as the existence of a unique full-rank fixed point or the fact that the Kraus operators corresponding to some number of applications of the channel span the full space of matrices. This will allow us to derive a quantum Wielandt's inequality for primitive quantum channels,

$$
q \leq\left(D^{2}-d+1\right) D^{2}
$$

where $D$ is the dimension of the Hilbert space, and $d$ the number of linearly independent Kraus operators. We will see that, under certain generic conditions on the Kraus operators, better inequalities can be derived. Finally, we apply the new inequalities to three problems related to channel capacities and to quantum spin chains: we derive a dichotomy theorem for the zero-error capacity of quantum channels and prove a conjecture for ground states of frustration-free spin Hamiltonians. Moreover, we show that our result also has new implications concerning the interaction-range of Hamiltonians with MPS as unique ground states [6].

\section{BASIC NOTIONS}

Let us start by fixing the notation and introducing the basic notions. We will consider quantum channels, i.e., TPCPMs, $\mathcal{E}$ : $\mathcal{M}_{D \times D} \rightarrow \mathcal{M}_{D \times D}$, where $\mathcal{M}_{D \times D}$ is the space of complex $D \times D$ matrices. Let us denote by $\mathcal{E}_{A}$ the quantum channel with Kraus operators $\left\{A_{k} \in \mathcal{M}_{D \times D}\right\}_{k=1}^{d}$, i.e.,

$$
\mathcal{E}_{A}(X)=\sum_{k=1}^{d} A_{k} X A_{k}^{\dagger} .
$$

We define $S_{n}(A) \subseteq \mathcal{M}_{D \times D}$ as the linear space spanned by all possible products of exactly $n$ Kraus operators, $A_{k_{1}} A_{k_{2}} \ldots A_{k_{n}}$, and by $A_{k}^{(n)}$ the elements of $S_{n}(A)$. There is a one-to-one correspondence between a quantum channel $\mathcal{E}$ and its Choi matrix $\omega(\mathcal{E}):=$ (id $\otimes$ $\mathcal{E})(\Omega)$ where $\Omega=\sum_{i, j=1}^{D}|i i\rangle\langle j j|$. It is readily verified that $\operatorname{rank}\left[\omega\left(\mathcal{E}_{A}{ }^{n}\right)\right]=\operatorname{dim}\left[S_{n}(A)\right]$. We further define $H_{n}(A, \varphi):=S_{n}(A)|\varphi\rangle \subseteq \mathbb{C}^{D}$ as the space spanned by all vectors $A_{k_{1}} A_{k_{2}} \ldots A_{k_{n}}|\varphi\rangle$, where $|\varphi\rangle \in \mathbb{C}^{D}$. That is, $\operatorname{rank}\left[\mathcal{E}_{A}{ }^{n}(|\varphi\rangle\langle\varphi|)\right]=\operatorname{dim}\left[H_{n}(A, \varphi)\right]$.

We introduce now three properties which will later turn out to be equivalent:

(a) A quantum channel $\mathcal{E}_{A}$ is called primitive if there exists some $n \in \mathbb{N}$ such that for all $|\varphi\rangle \in \mathbb{C}^{D}$, $H_{n}(A, \varphi)=\mathbb{C}^{D}$. In other words, if for every input density operator $\rho$ the output $\mathcal{E}_{A}{ }^{n}(\rho)$ obtained after $n$ applications of the channel has full-rank. We will denote by $q\left(\mathcal{E}_{A}\right)$ the minimum $n$ for which that condition is fulfilled. Note that if $\mathcal{E}_{A}$ is primitive, then for every $m \in \mathbb{N}, \mathcal{E}_{A}^{m}$ is primitive, too, and we have $H_{n}(A, \varphi)=\mathbb{C}^{D}$ for all $n \geq q\left(\mathcal{E}_{A}\right)$. 
(b) A quantum channel $\mathcal{E}_{A}$ is said to have eventually full Kraus rank if there exists some $n \in \mathbb{N}$ such that $S_{n}(A)=\mathcal{M}_{D \times D}$, i.e., if $\operatorname{rank}\left[\omega\left(\mathcal{E}_{A}{ }^{n}\right)\right]=D^{2}$. We denote by $i(A)$ the minimum $n$ for which that condition is satisfied. Obviously, if $\mathcal{E}_{A}$ fulfills this property, then $S_{n}(A)=\mathcal{M}_{D \times D}$ for all $n \geq i(A)$.

(c) We say that a quantum channel $\mathcal{E}_{A}$ is strongly irreducibl 1 if the following two conditions are fulfilled: (i) $\mathcal{E}_{A}$ has a unique eigenvalue, $\lambda$, with $|\lambda|=1$; (ii) the corresponding eigenvector, $\rho$, is a positive definite operator $(\rho>0)$. This implies the convergence

$$
\lim _{n \rightarrow \infty} \mathcal{E}_{A}^{n}=\mathcal{E}_{A}^{\infty},
$$

where $\mathcal{E}_{A}^{\infty}(X):=\rho \operatorname{tr}(X)$. Note that, for instance, the generalized Frobenius theorem proved in [7, Theorem 2.5] ensures that a TPCPM always has an eigenvalue $\lambda=1$ with eigenvector $\rho \geq 0$. In this case it was already known [8, Lemma 5.2] that there exits an upper bound for $i(A)$ related with the second eigenvalue $\lambda_{2}$ of $\mathcal{E}_{A}$, which is essentially $i(A) \lesssim O\left(\exp \frac{1}{\lambda_{2}}\right)$.

Our first simple observation is that (b) implies (a), or stated quantitatively:

Proposition 1. For every quantum channel $\mathcal{E}_{A}$ we have that $q\left(\mathcal{E}_{A}\right) \leq i(A)$.

Proof: Take any $n \geq i(A)$. Then, by the definition of $i(A)$, the Choi matrix $\omega\left(\mathcal{E}_{A}^{n}\right)$ has full-rank, so that

$$
\mathcal{E}_{A}^{n}(|\varphi\rangle\langle\varphi|)=(\mathbb{1} \otimes\langle\bar{\varphi}|) \omega\left(\mathcal{E}_{A}^{n}\right)(\mathbb{1} \otimes|\bar{\varphi}|)
$$

also has full-rank.

Before continuing the analysis of the relationships among the three properties above in the quantum context, let us connect them to the classical notion of primitivity. Given a stochastic matrix $A=\left(a_{i j}\right)$, let us consider the map $\mathcal{E}_{A}$ defined by the Kraus operators $A_{i, j}=\sqrt{a_{i, j}}|i\rangle\langle j| . \mathcal{E}_{A}$ has the property that for an operator $\rho$ with entries $\rho_{i, j}=\delta_{i, j} p_{i} \geq 0$, $\rho^{\prime}:=\mathcal{E}_{A}(\rho)$ is diagonal with $\rho_{i, j}^{\prime}=\delta_{i, j} p_{i}^{\prime}$, with $p^{\prime}=A p$. Thus, $\mathcal{E}_{A}$ implements the stochastic map $A$, i.e., the quantum channel reduces to the classical channel when applied to diagonal density operators. Note that $d$ is the number of positive entries of the stochastic matrix in the classical case, so the general quantum bound applied to a classical channel is always worse than the classical bound.

Let us consider $A$ primitive and denote by $p(A)$ its classical index of primitivity. Then, we have:

Proposition 2. Let us consider a primitive stochastic map $A$ and the corresponding $T P C P M \mathcal{E}_{A}$. Then, $\mathcal{E}_{A}$ is also primitive and the equality $q(A)=p(A)=i(A)$ holds.

Proof: It is clear that $p(A) \leq q\left(\mathcal{E}_{A}\right)$ and we proved in Prop.11 that $q\left(\mathcal{E}_{A}\right) \leq i(A)$. In order to show that $i(A) \leq p(A)$,

\footnotetext{
${ }^{1}$ The notion of 'irreducibility', used for instance in [7], differs from our definition of 'strong irreducibility' by allowing for other eigenvalues of magnitude one. In fact, $\mathcal{E}$ is strongly irreducible iff $\mathcal{E}^{n}$ is reducible for all $n \in \mathbb{N}$. This property is known as injectivity in the context of Matrix Product States.
}

we define $\tilde{A}_{i, j}=\sqrt{a_{i, j}} A_{i, j}, n=p(a)-1$, and take

$$
\sum_{k_{1}, \ldots, k_{n}=1}^{D} \tilde{A}_{i, k_{1}} \tilde{A}_{k_{1}, k_{2}} \ldots \tilde{A}_{k_{n}, j}=\left(A^{p(a)}\right)_{i, j}|i\rangle\langle j| \neq 0 .
$$

Thus, $|i\rangle\langle j| \in S_{p(A)}(A)$ for all $i, j$.

We note that $q\left(\mathcal{E}_{A}\right)$ is different from $i(A)$ in the general case. To see that, let us consider an example with $d=3$, $D=2$, and take as Kraus operators $\sigma_{\alpha} / \sqrt{3}$, where $\alpha=x, y, z$ labels the three Pauli matrices. Here $q\left(\mathcal{E}_{A}\right)=1<i(A)=2$.

However, the following proposition shows that $i(A)$ is finite whenever $q\left(\mathcal{E}_{A}\right)$ is. In fact, all three definitions above are equivalent:

Proposition 3. Given a quantum channel $\mathcal{E}_{A}$, the following statements are equivalent: (a) $\mathcal{E}_{A}$ is primitive; (b) $\mathcal{E}_{A}$ has eventually full Kraus rank; (c) $\mathcal{E}_{A}$ is strongly irreducible.

Proof: We denote by $\rho \geq 0$ an eigenoperator of $\mathcal{E}_{A}$ corresponding to the eigenvalue $\lambda=1$.

(b) $\Rightarrow$ (a)

This implication is given by Prop 1

(a) $\Rightarrow$ (c)

We prove it by contradiction. Let us assume that $\mathcal{E}_{A}$ is not strongly irreducible. Then, we must have at least one of the following cases: (i) $\rho$ is not full-rank; (ii) there exists another eigenoperator, $\rho^{\prime}$, corresponding to $\lambda=1$; (iii) there is another eigenvalue, $\lambda^{\prime}$, with $\left|\lambda^{\prime}\right|=1$. Since for all $n \in N, \mathcal{E}_{A}^{n}(\rho)=\rho$, (i) automatically implies that $\mathcal{E}_{A}$ is not primitive. Furthermore, if we have (ii), choosing $\epsilon=1 / \max \left[\operatorname{spec}\left(\rho^{-1 / 2} \rho^{\prime} \rho^{-1 / 2}\right)\right]$ we have that $\tilde{\rho}=\rho-\epsilon \rho^{\prime} \geq 0$ is not full-rank and thus we are back in (i). Moreover, it is proven in the demonstration of [8, Proposition 3.3] that, if (i) and (ii) do not hold, the other possible eigenvalues of modulus 1 are the $p$-th roots of unity for some finite $p \in \mathbb{N}$. Therefore, we have (ii) for $\mathcal{E}_{A}^{p}$, and thus $\mathcal{E}_{A}^{p}$ cannot be primitive.

(c) $\Rightarrow$ (b)

This implication can be deduced from [8, Lemma 5.2], but we include here a proof for completeness. We prove it by contradiction. Let us assume that $\mathcal{E}_{A}$ is (j) strongly irreducible, but (jj) does never get full Kraus rank. If we have (j) then $\rho$ is full-rank and Eq. (2) is fulfilled. Because of (jj), for all $n \in \mathbb{N}$ and $A_{k}^{(n)} \in S_{n}(A)$, there exists some $B_{n} \neq 0$ such that $\operatorname{tr}\left(A_{k}^{(n)} B_{n}\right)=0$. Thus,

$$
\begin{gathered}
\left|\operatorname{tr}\left(\rho B_{n}^{\dagger} B_{n}\right)\right|=\left.\left|\sum_{k_{1}, \ldots, k_{n}}\right| \operatorname{tr}\left(A_{k_{1}} \cdots A_{k_{n}} B_{n}\right)\right|^{2}-\operatorname{tr}\left(\rho B_{n}^{\dagger} B_{n}\right) \mid \\
=\left|\operatorname{tr}\left[\Omega\left(\mathcal{E}_{A}^{n} \otimes \mathrm{id}\right)\left(\tilde{B}_{n} \Omega \tilde{B}_{n}^{\dagger}\right)\right]-\operatorname{tr}\left[\Omega\left(\mathcal{E}_{A}^{\infty} \otimes \mathrm{id}\right)\left(\tilde{B}_{n} \Omega \tilde{B}_{n}^{\dagger}\right)\right]\right| \\
\leq c_{n}\|\Omega\|_{\infty} \operatorname{tr}\left(\tilde{B}_{n} \Omega \tilde{B}_{n}^{\dagger}\right)=D c_{n} \operatorname{tr}\left(B_{n}^{\dagger} B_{n}\right)
\end{gathered}
$$

where $\tilde{B}_{n}=B_{n} \otimes \mathbb{1}$ and $\lim _{n} c_{n}=0$. If $\rho$ was full-rank, then for all $X \geq 0$ one would have

$$
\operatorname{tr}(\rho X) \geq \frac{1}{\left\|\rho^{-1}\right\|_{\infty}} \operatorname{tr}(X)
$$

and we obtain a contradiction. 
As a consequence of Prop. 3, we obtain that primitivity of a quantum channel can be decided by observing its spectral properties. In fact, this is the precise quantum analogue of the classical result that a stochastic matrix is primitive iff it has a unique eigenvalue of maximum modulus and a positive definite fixed point (cf. [1]).

\section{QUANTUM WIELANDT'S INEQUALITIES}

In order to reach a quantum version of Wielandt's inequality, i.e. bounds for $q\left(\mathcal{E}_{A}\right)$ and $i(A)$, we require some preliminary lemmas:

Lemma 1. Let $\mathcal{E}_{A}$ be a primitive quantum channel on $\mathcal{M}_{D \times D}$ with $d$ Kraus operators. Then, there is a $A^{(n)} \in S_{n}(A)$ with $n \leq D^{2}-d+1$ such that $\operatorname{tr}\left(A^{(n)}\right) \neq 0$.

Proof: Let us denote by $T_{n}(A)$ the span of all $S_{m}(A)$ with $m \leq n$. We just have to show that: (*) for any $n \in$ $\mathbb{N}$, if $\operatorname{dim}\left[T_{n}(A)\right]<D^{2}$, then $\operatorname{dim}\left[T_{n+1}(A)\right]>\operatorname{dim}\left[T_{n}(A)\right]$. Since $\operatorname{dim}\left[T_{1}(A)\right]=d$, by iteration we obtain that $T_{D^{2}-d+1}=$ $\mathcal{M}_{D \times D}$. This implies that a linear combination of the elements of $S_{n}(A)$ with various $n \leq D^{2}-d+1$ must be equal to the identity, and thus at least one of the elements must have nonzero trace. To prove (*) we note that, by definition, $T_{n}(A) \subseteq$ $T_{n+1}(A)$. If they would be equal, then $T_{m}(A)=T_{n}(A)$ for all $m>n$. Thus, $\operatorname{dim}\left[T_{n}(A)\right]=D^{2}$ since otherwise the map $\mathcal{E}_{A}$ would not be primitive.

Lemma 2. Let $\mathcal{E}_{A}$ be primitive such that $A_{1}|\varphi\rangle=\mu|\varphi\rangle$ with $\mu \neq 0$. Then: (a) $H_{D-1}(A, \varphi)=\mathbb{C}^{D}$. (b) If $A_{1}$ is not invertible, then for all $|\psi\rangle \in \mathbb{C}^{D},|\varphi\rangle\langle\psi| \in S_{D^{2}-D+1}(A)$;

Proof: (a) We define $K_{n}(A, \varphi)$ as the span of all $H_{m}(A, \varphi)$ with $m \leq n$ together with $|\varphi\rangle$. If $\operatorname{dim}\left[K_{n}(A, \varphi)\right]<$ $D$, then $\operatorname{dim}\left[K_{n+1}(A, \varphi)\right]>\operatorname{dim}\left[K_{n}(A, \varphi)\right]$, since otherwise the map would not be primitive. Thus, $K_{D-1}(A, \varphi)=\mathbb{C}^{D}$. That is, for all $|\phi\rangle \in \mathbb{C}^{D}$, there exist matrices $A^{(n)} \in S_{k_{n}}(A)$, $k_{n} \leq D-1$ such that (with $A^{(0)} \propto \mathbb{1}$ )

$$
|\phi\rangle=\sum_{n=0}^{D-1} A^{(n)}|\varphi\rangle=\sum_{n=0}^{D-1} A^{(n)} \frac{A_{1}^{D-k_{n}}}{\mu^{D-k_{n}}}|\varphi\rangle,
$$

and thus, $|\phi\rangle \in H_{D-1}(A, \varphi)$. (b) We write $A_{1}$ in the Jordan standard form and divide it into two blocks. The first one, of size $\tilde{D} \times \tilde{D}$, consists of all Jordan blocks corresponding to non-zero eigenvalues, whereas the second one contains all those corresponding to zero eigenvalues. We denote by $P$ the projector onto the subspace where the first block is supported and by $r \leq D-\tilde{D}$ the size of the largest Jordan block corresponding to a zero eigenvalue. We have

$$
A_{1} P=P A_{1}, \quad A_{1}^{r}=A_{1}^{r} P .
$$

We define $R_{n}(A)=P S_{n}(A)$ and show that $R_{D \tilde{D}}(A)=$ $\mathcal{M}_{\tilde{D} \times D}$. For all $n \in \mathbb{N}$, $\operatorname{dim}\left[R_{n+1}(A)\right] \geq \operatorname{dim}\left[R_{n}(A)\right]$. The reason is that for any linearly independent set of matrices $A_{k}^{(n)} \in R_{n}(A), A_{1} A_{k}^{(n)} \in R_{n+1}(A)$ are also linearly independent, given that $A_{1}$ is invertible on its range. By following the reasoning of [6. Appendix A] we get that, if $\operatorname{dim}\left[R_{n+1}(A)\right]=$ $\operatorname{dim}\left[R_{n}(A)\right]=: D^{\prime}$, then $\operatorname{dim}\left[R_{m}(A)\right]=D^{\prime}$ for all $m>n$, which is incompatible with $\mathcal{E}_{A}$ being primitive unless $D^{\prime}=$
$\tilde{D} D$. Thus, for all $|\psi\rangle \in \mathbb{C}^{D}$, there exists $A \in S_{\tilde{D} D}$ with $|\varphi\rangle\langle\psi|=P A=A_{1}^{r} P A / \mu^{r}=A_{1}^{r} A / \mu^{r}=A^{\prime} \in S_{\tilde{D} D+r}$. By using that $\tilde{D} \leq D-r$ and that $r \geq 1$ (since $A_{1}$ is supposed to be not invertible) we get $\tilde{D} D+r \leq D^{2}-D+1$, which concludes the proof.

We have now the necessary tools to prove our main result.

Theorem 1. Let $\mathcal{E}_{A}$ be a primitive quantum channel on $\mathcal{M}_{D \times D}$ with $d$ Kraus operators. Then $q\left(\mathcal{E}_{A}\right) \leq i(A)$ and

1) in general $i(A) \leq\left(D^{2}-d+1\right) D^{2}$,

2) if the span of Kraus operators $S_{1}(A)$ contains an invertible element, then $i(A) \leq D^{2}-d+1$,

$3)$ if the span of Kraus operators $S_{1}(A)$ contains a noninvertible element with at least one non-zero eigenvalue, then $i(A) \leq D^{2}$.

Proof: The inequality $q\left(\mathcal{E}_{A}\right) \leq i(A)$ is shown in Prop. 1 2. If there is an invertible element, then it follows from [6] Appendix A, Proposition 2] that $\operatorname{dim} S_{n+1}(A)>\operatorname{dim} S_{n}(A)$ until the full matrix space $\mathcal{M}_{D \times D}$ is spanned and thus $i(A) \leq$ $D^{2}-d+1$.

1. Let us denote by $\left\{A_{k}^{(n)}\right\}$ the Kraus operators corresponding to $\mathcal{E}_{A}^{n}$. According to Lemma 1, one of them, say $A_{1}^{(n)}$, has non-zero trace and therefore there exists $|\varphi\rangle$ such that $A_{1}^{(n)}|\varphi\rangle=\mu|\varphi\rangle$ with $\mu \neq 0$. If $A_{1}^{(n)}$ is invertible, then 1 . is implied by 2 ., so we can assume that it is not invertible. According to Lemma 2.(b), for all $|\psi\rangle,|\chi\rangle \in \mathbb{C}^{D}$ we have $|\varphi\rangle\langle\psi| \in S_{D^{2}-D+1}(B)$; and according to Lemma 2](a) $|\chi\rangle\langle\psi| \in S_{D^{2}}(B)=S_{n D^{2}}(A)$. This implies that $S_{n D^{2}}(A)=M_{D \times D}$ and hence the general bound 1. follows.

The argument which proves 3 . is completely analogous. The main difference is that, in order to guarantee the existence of a Kraus operator with non-zero eigenvalue, we have to apply Lemma 1 for the general case 1 . and to take the $n$ 'th power of the quantum channel for some $n \leq D^{2}-d+1$.

We do not know whether, or in which cases, our bounds are sharp. A simple lower bound to $i(A)$ comes from the examples showing that the classical Wielandt's inequality is sharp. In these cases $q\left(\mathcal{E}_{A}\right)=i(A)=D^{2}-2 D+2$. A lower bound that goes beyond this value is given by the next example.

Example 1. Let us consider the completely positive map described by the following Kraus operators $A_{i} \in \mathcal{M}_{D}$ : $A_{0}=\sum_{i=0}^{D-1}|i+1\rangle\langle i|$ and $A_{1}=|1\rangle\langle D-1|$, with $|D\rangle=|0\rangle$. In this case $i(A)=D^{2}-D$ which is larger than the bound appearing in Wielandt's classical inequality whenever $D>2$.

Proof: Consider the case $D>2$ as $D=2$ is readily verified by inspection. Then $A_{1}^{2}=0$ and $A_{1} A_{0}^{k} A_{1}=A_{1} \delta_{k, D-2}$. Therefore

$$
S_{N}(A)=\operatorname{span}\left\{A_{0}^{N}, A_{0}^{k} A_{1} A_{0}^{l}\right\}
$$

where $k, l=0, \ldots, D-1$ fulfill the additional constraint that

$$
k+l+1+n(D-1)+m D=N
$$

for some $n, m \in \mathbb{N}_{0}$. The additional constraint comes from the fact that $A_{1}$ can stem from $A_{1} A_{0}^{D-2} A_{1}$ or $A_{1} A_{0}^{D-2} A_{1} A_{0}^{D-2} A_{1}$ etc. which is a monomial of degree $1+n(D-1)$. The fact that $A_{0}^{m D}=\mathbb{1}$ is taken care of by the additional factor $m D$. Now assume that $N=D(D-1)-1$. Let 
us upper bound the number of linearly independent operators in $S_{N}(A)$. Clearly, for every chosen $n$ and $k$, we get that $l$ and $m$ are fixed by the additional constraint. For $n=D-1$, the range of $k$ is by Eq. (7) restricted to $k=0, \ldots, D-3$. So in total we have at most $(D-2)+(D-1) D+1=D^{2}-1$ independent elements which cannot span the entire matrix algebra. Thus $i(A) \geq D^{2}-D$ (if the map is primitive). That this bound is sharp, and the map actually primitive, is seen by noting that for $N=D^{2}-D$ the constraint in Eq. (7) allows us to choose $k$ and $l$ freely by adjusting $n$ and $m$. Then, however, $A_{0}^{k} A_{1} A_{0}^{l}$ runs through all matrix units which span the entire matrix algebra.

We also note that for small dimension $D=2,3$ there is always an element in $S_{1}(A)$ which has a non-zero eigenvalue. In other words, in these cases the first bound in Thm 1 never applies without one of the other bounds. The fact that $S_{1}(A)$ has this property for $D=2,3$ stems from the classification of nilpotent subspaces [9]: assume that $S_{1}(A)$ would be a nilpotent subspace within the space of $D \times D$ matrices. Then for $D=2$ its dimension would have to be one, so it could not arise from the Kraus operators of a quantum channel. Similarly, for $D=3$ there are (up to similarity transformations) two types of nilpotent subspaces [9] with $d>1$ : one of dimension $d=3$, the space of uppertriangular matrices, whose structure does not allow the tracepreserving property, and one of dimension $d=2$ which only leads to quantum channels having a (in modulus) degenerate largest eigenvalue. Hence, if $S_{1}(A)$ is generated by the Kraus operators of a primitive quantum channel, then it cannot be nilpotent if $D=2,3$.

In the following we will show some applications of the derived bounds.

\section{ZERO-ERROR CAPACITY}

The zero error capacity $C_{0}$ of a noisy channel was defined by Shannon in [10] as follows: There exists a sequence of codes of increasing block length such that the rate of transmission approaches $C_{0}$ and the probability of error after decoding is zero (instead of approaches zero as in the definition of the usual capacity). Furthermore, this is not true for any value higher than $C_{0}$. This concept becomes important in situations where no error can be tolerated or when a fixed finite number of uses of the channel is available and it constitutes a central topic in information theory [11]. The definition can be translated straightforwardly to the case of quantum channels [12], where a number of interesting results appear: the computation of this is QMA-hard [13] and it can be superactivated [14] (see also [15], [16]).

We will show here a dichotomy behavior for the power of a quantum channel with full-rank fixed point (e.g., a unital quantum channel) as a consequence of our quantum Wielandt's inequality. If we think of the power $\mathcal{E}^{n}$ as a channel describing the input-output relation after $n$ units in time/space, then the subsequent result shows that there is a critical time/length $n=$ $q\left(\mathcal{E}_{A}\right)$ such that a successful transmission through $\mathcal{E}^{n}$ implies the possibility of a successful transmission to arbitrary $m \geq n$. By the quantum Wielandt's inequality, this critical value can be taken $\left(D^{2}-d+1\right) D^{2}$ and is therefore universal. It depends only on $D$ and not on the channel itself.

Theorem 2. If $\mathcal{E}$ is a quantum channel with a full-rank fixed point, and we call $C_{0}(\mathcal{E})$ the 0 -error-classical capacity of $\mathcal{E}$. Then, either ${ }^{2} C_{0}\left(\mathcal{E}^{n}\right) \geq 1$ for all $n$ or $C_{0}\left(\mathcal{E}^{q(\mathcal{E})}\right)=0$.

Proof: We split the problem into two cases:

Case 1:

Let us assume that the channel has two (or more) different fixed points. By following [17], the set of fixed points of a quantum channel which has a fullrank fixed point is of the form $V\left(\oplus_{i} \rho_{i} \otimes M_{m_{i}}\right) V^{\dagger}$ where $V$ is some unitary, the $\rho_{i}$ 's are density matrices with full-rank, and $M_{m_{i}}$ is a full matrix algebra of dimension $m_{i}$. Consequently, if the direct sum is non-trivial, we can encode a classical bit in the corresponding projectors. If the direct sum is trivial, then the space of matrices is non-trivial, i.e., there is a $m_{i} \geq 2$, and we can encode one qubit in it. In either case $C_{0}\left(\mathcal{E}^{n}\right) \geq 1$ independent of $n$.

Similar statements hold if the channel has only one fixed point (which is by assumption full-rank) but another eigenvalue $\mu$ of magnitude one: since $\mu$ is a root of unity, i.e., there is an integer $p \leq D^{2}$ with $\mu^{p}=1$, we have that $\mathcal{E}^{p}$ has several fixed points. So again we can safely encode a bit and $C_{0}\left(\mathcal{E}^{n}\right) \geq 1$ independent of $n$.

Case 2:

If the channel has just one fixed point and no other eigenvalue of magnitude one, then it is primitive by Prop 3. So $\mathcal{E}^{q(\mathcal{E})}$ has the property that all output states are full-rank. This implies [14] that $C_{0}\left(\mathcal{E}^{n}\right)=$ 0 for all $n \geq q(\mathcal{E})$.

\section{Frustration-FreE Hamiltonians And Matrix PRODUCT STATES}

Matrix Product States have proven to be a useful family of quantum states for explaining the low energy sectors of locally interacting one-dimensional systems. They constitute a suitable variational ansatz for instance to compute ground state energies to high accuracy [18] which can be explained by the fact that MPS approximate ground states of local 1D Hamiltonians well [19]. Similarly they are used to understand effects on 1D quantum systems on analytic grounds, such as string orders [20], symmetries [21], renormalization flows [22] or sequential interactions [23], [24].

Associated to each translational invariant MPS of the form

$$
\left|\psi_{A}\right\rangle=\sum_{i_{1}, \ldots, i_{N}} \operatorname{tr}\left(A_{i_{1}} \cdots A_{i_{N}}\right)\left|i_{1} \cdots i_{N}\right\rangle
$$

there is a parent Hamiltonian $H_{A}$ which is frustration-free and has $\left|\psi_{A}\right\rangle$ as ground state. Let us start by defining the concept of frustration-free Hamiltonian. Consider a local translational invariant Hamiltonian in a spin chain $H=\sum_{i} \tau^{i}(h)$ where

\footnotetext{
${ }^{2}$ In fact, one can consider here even the one-shot zero-error capacity, that is, the one obtained with a single use of the channel.
} 
$h$ denotes the local interaction term and $\tau$ the translation operator. Then,

Definition 3. The Hamiltonian is called frustration-free if its ground state $\left|\psi_{0}\right\rangle$ minimizes the energy locally, that is, if

$$
\min _{|\psi\rangle}\langle\psi|\mathbb{1} \otimes h| \psi\rangle=\left\langle\psi_{0}|\mathbb{1} \otimes h| \psi_{0}\right\rangle
$$

We assume w.l.o.g. that (9) is equal to 0. Such Hamiltonians include classical Hamiltonians, where the terms commute, as well as all parent Hamiltonians appearing in the Matrix Product State (MPS) theory [6], [8], [25]. A remarkable example is the AKLT Hamiltonian [26].

The corresponding local interaction term $h$ above is constructed as the projector onto the orthogonal complement of the image of

$$
X \in M_{D \times D} \mapsto \sum_{i_{1}, \ldots, i_{L}} \operatorname{tr}\left(X A_{i_{1}} \cdots A_{i_{L}}\right)\left|i_{1} \cdots i_{L}\right\rangle,
$$

for some sufficiently large interaction range $L$. Note that the map in Eq. 10 is injective for sufficiently large $L$ iff the map $\mathcal{E}_{A}(X):=\sum_{i} A_{i} X A_{i}^{\dagger}$ is primitive 3 and that injectivity holds for all $L \geq i(A)$. The following theorem which was proven in [6], [8] provides another application for the quantum Wielandt's inequalities for $i(A)$ :

Theorem 4. If the interaction range $L$ of the parent Hamiltonian $H_{A}$ satisfies $L>i(A)$, then the MPS $\left|\psi_{A}\right\rangle$ is the unique ground of $H_{A}$, and $H_{A}$ has a spectral gap above the ground state energy.

Hence, the quantum Wielandt's inequality provides a bound for the interaction length required to get a good parent Hamiltonian for a MPS. Indeed, the existence of such inequality was already conjectured in the context of MPS [6, Conjectures 1 and 2] and some results obtained so far about MPS do directly depend on the validity of that conjecture. In particular, a dichotomy result for ground states of frustrationfree Hamiltonians, sketched in [6] and for which we give a complete proof below, and the characterization of the existence of global symmetries in arbitrary MPS given in [21].

One might conjecture that the ground state of every frustration-free Hamiltonian (with non-degenerate ground state) is a MPS. In fact, the quantum Wielandt's inequality allows us to get a dichotomy theorem in this direction:

Theorem 5. Take a local term $h$ with interaction length $L$ and assume that $H_{N}=\sum_{i=1}^{N} \tau^{i}(h)$ is frustration-free and has a unique ground state for every $N$. Its ground state can be represented as an MPS with matrix size $D \times D$, where $D$ is

(i) either independent of $N$,

(ii) or $>\Omega\left(N^{\frac{1}{5}}\right)$ for all prime numbers $N$.

Proof: Let us recall from [6, Theorem 5] that each MPS with $D<N$ and $N$ prime can be mapped into a canonical decomposition where all matrices are block diagonal $A_{i}=\oplus_{j=1}^{b} A_{i}^{j}$ and each block satisfies injectivity. Moreover, [6. Theorem 11] states that if $b \geq 2, L_{0}=\max _{j} i\left(A^{j}\right)$ and

\footnotetext{
${ }^{3} \mathcal{E}_{A}$ may be assumed to be trace-preserving without loss of generality [6].
}

$L$ is the interaction length of any frustration-free translational invariant Hamiltonian $H$ on $N$ spins having $\left|\psi_{A}\right\rangle$ as ground state, the condition $N \geq 3(b-1)\left(L_{0}+1\right)+L$ implies that $\left|\psi_{A^{j}}\right\rangle$ is also a ground state of $H$ for all $j$. Since the quantum Wielandt's inequality allows us to bound $L_{0} \leq O\left(D^{4}\right)$ and trivially $b \leq D$, we get that either (ii) $D \geq \Omega\left(N^{\frac{1}{5}}\right)$, or $b=1$ and $\operatorname{ker}(h) \ni \sum_{i_{1}, \cdots, i_{L}} \operatorname{tr}\left(X A_{i_{1}} \cdots A_{i_{L}}\right)\left|i_{1} \cdots i_{L}\right\rangle$ where $X \in S_{N-L}(A)$. Since by the quantum Wielandt's inequality again $N-L \geq i(A)$, we get that $\operatorname{ker}(h) \supseteq$ $\left\{\sum_{i_{1}, \cdots, i_{L}} \operatorname{tr}\left(X A_{i_{1}} \cdots A_{i_{L}}\right)\left|i_{1} \cdots i_{L}\right\rangle: X \in M_{D \times D}\right\}$. This trivially implies that $\left|\psi_{A}\right\rangle$ is also a ground state for $H_{N^{\prime}}$ when $N^{\prime}>N$ and therefore the only one, so we obtain (i).

Regarding the restriction to prime $N$, note that by the Prime Number Theorem the number of primes less than or equal to a given $N$ is asymptotically $\frac{N}{\log N}$. Therefore in (ii) there are many lengths for which there is no MPS representation of the ground state with small matrices.

\section{CONCLUSIONS AND OPEN PROBLEMS}

The present work focuses on finding dimension dependent bounds for the number of times that a quantum channel has to be applied in order to have a full-rank Choi matrix. Once this is obtained, bounds on the quantum index of primitivity $q$ are straightforwardly achieved, since $q \leq i(A)$. As direct applications of these results, we derive dichotomy theorems for the zero-error capacity of quantum channels as well as a couple of results in Matrix Product States theory. The first one is the demonstration of a conjecture with interesting implications for ground states of frustration-free Hamiltonians and the other a theorem which introduces new implications concerning the interaction-range of Parent Hamiltonians.

As a possible future research, we suggest that it might be advantageous to focus on computing bounds for $q$ directly, since $q \neq i(A)$ in general. This is interesting because, while some applications (like the ones in the MPS context) require bounds on $i(A)$, others like Thm. 2 are based on $q$. For instance, from a purely mathematical point of view, $i(A)$ is not applicable for positive maps (the usual framework of Frobenius theory), unless the map is completely positive. Furthermore, we leave open the question about optimal bounds for both $q$ and $i(A)$.

Another possible future research is to relate the quantum Wielandt's inequality to graph theory and quantum random walks. In the classical case, there is a close relationship between stochastic matrices and graph theory (by taking $A$ the adjacency matrix of a graph) which makes the inequality broadly applicable. In fact, the usual proofs of the classical inequality are based on the graph picture. However, although there are different attempts to establish a relationship between quantum channels and quantum graphs [27], [28], there is not any well-defined analogous one for the quantum context.

\section{ACKNOWLEDGMENT}

The authors would like to thank Frank Verstraete for his useful suggestions and discussions and A. Nogueira for the invaluable technical assistance. 


\section{REFERENCES}

[1] R. A. Horn and C. R. Johnson, Topics in Matrix Analysis. Cambridge University Press, 1991.

[2] H. Wielandt, "Unzerlegbare, nicht negative Matrizen," Mathematische Zeitschrift, vol. 52, no. 1, pp. 642-648, Dec 1950.

[3] E. Seneta, Non-negative matrices and Markov chains, ser. Springer Series in Statistics. Springer-Verlag Berlin Heidelberg, 2006.

[4] J. L. R. Alfonsín, The Diophantine Frobenius Problem, ser. Oxford Lecture Series in Mathematics and its applications. Oxford University Press, 2005, vol. 30.

[5] R. S. Varga, Matrix Iterative Analysis, ser. Springer Series in Computational Mathematics. Springer-Verlag Berlin Heidelberg, 2000, vol. 27.

[6] D. Pérez-García, F. Verstraete, M. Wolf, and J. Cirac, "Matrix product state representations," Quantum Inf. Comput., vol. 7, p. 401, 2007.

[7] D. E. Evans and R. Hoegh-Krohn, "Spectral properties of positive maps on c*-algebras," Journal of the London Mathematical Society, vol. s2-17, no. 2, pp. 345-355, Apr 1978.

[8] M. Fannes, B. Nachtergaele, and R. F. Werner, "Finitely correlated states on quantum spin chains." Commun. Math. Phys., vol. 144, no. 3, pp. 443-490, 1992.

[9] M. A. Fasoli, "Classification of nilpotent linear spaces in M(4; C)," Communications in Algebra, vol. 25, no. 6, pp. 1919 - 1932, 1997.

[10] C. Shannon, "The zero error capacity of a noisy channel," IRE Trans. Inform. Theory, vol. 2, no. 3, pp. 8-19, Sep 1956.

[11] J. Korner and A. Orlitsky, "Zero-error information theory," IEEE Trans. Inform. Theory, vol. 44, no. 6, pp. 2207-2229, Oct 1998.

[12] R. A. C. Medeiros and F. M. de Assis, "Quantum zero-error capacity," Int. J. Quant. Inf., vol. 3, no. 1, pp. 135-139, 2005.

[13] S. Beigi and P. W. Shor, "On the complexity of computing zero-error and Holevo capacity of quantum channels," Sep 2007. [Online]. Available: http://arxiv.org/abs/0709.2090v3

[14] T. S. Cubitt, J. Chen, and A. W. Harrow, "Superactivation of the asymptotic zero-error classical capacity of a quantum channel," Jun 2009. [Online]. Available: http://arxiv.org/abs/0906.2547v2

[15] R. Duan and Y. Shi, "Entanglement between two uses of a noisy multipartite quantum channel enables perfect transmission of classical information," Phys. Rev. Lett., vol. 101, no. 2, p. 020501, Jul 2008.

[16] R. Duan, "Super-activation of zero-error capacity of noisy quantum channels," Jun 2009. [Online]. Available: http://arxiv.org/abs/0906.2527v1

[17] G. Lindblad, "A general no-cloning theorem," Letters in Mathematical Physics, vol. 47, no. 2, pp. 189-196, Jan 1999.

[18] F. Verstraete, V. Murg, and J. I. Cirac, "Matrix product states, projected entangled pair states, and variational renormalization group methods for quantum spin systems," Advances in Physics, vol. 57, no. 2, pp. $143-$ 224,2008

[19] M. B. Hastings, "An area law for one-dimensional quantum systems," Journal of Statistical Mechanics: Theory and Experiment, vol. 2007, no. 08, p. P08024, 2007.

[20] D. Pérez-García, M. M. Wolf, M. Sanz, F. Verstraete, and J. I. Cirac, "String order and symmetries in quantum spin lattices," Phys. Rev. Lett., vol. 100, no. 16, p. 167202, Apr 2008.

[21] M. Sanz, M. M. Wolf, D. Pérez-García, and J. I. Cirac, "Matrix product states: Symmetries and two-body hamiltonians," Phys. Rev. A, vol. 79, no. 4, p. 042308, Apr 2009.

[22] F. Verstraete, J. I. Cirac, J. I. Latorre, E. Rico, and M. M. Wolf, "Renormalization-group transformations on quantum states," Phys. Rev. Lett., vol. 94, no. 14, p. 140601, Apr 2005.

[23] C. Schön, E. Solano, F. Verstraete, J. I. Cirac, and M. M. Wolf, "Sequential generation of entangled multiqubit states," Phys. Rev. Lett., vol. 95 , no. 11, p. 110503, Sep 2005.

[24] L. Lamata, J. León, D. Pérez-García, D. Salgado, and E. Solano, "Sequential implementation of global quantum operations," Phys. Rev. Lett., vol. 101, no. 18, p. 180506, Oct 2008.

[25] N. Schuch, I. Cirac, and D. Pérez-García, "Peps as ground states: degeneracy and topology," Jan 2010. [Online]. Available: http://arxiv.org/abs/1001.3807v2

[26] I. Affleck, T. Kennedy, E. H. Lieb, and H. Tasaki, "Valence bond ground states in isotropic quantum antiferromagnets," Communications in Mathematical Physics, vol. 115, no. 3, pp. 477-528, Sep 1988.

[27] J. Kempe, "Quantum random walks: an introductory overview," Contemporary Physics, vol. 44, no. 4, pp. 307 - 327, 2003.

[28] A. Ben-Aroya and A. Ta-Shma, "Quantum expanders and the quantum entropy difference problem," 2007. [Online]. Available: http://arxiv.org/abs/quant-ph/0702129v3 